\title{
Fertility outcomes in asthma: a clinical study of 245 women with unexplained infertility
}

\author{
Elisabeth Juul Gade ${ }^{1,2}$, Simon Francis Thomsen ${ }^{3,4}$, Svend Lindenberg ${ }^{2}$ and \\ Vibeke Backer ${ }^{1}$
}

Affiliations: ${ }^{1}$ Respiratory Research Unit, Dept of Respiratory Medicine, Bispebjerg University Hospital, Copenhagen, Denmark. ${ }^{2}$ Copenhagen Fertility Center, Copenhagen, Denmark. ${ }^{3}$ Dept of Dermatology, Bispebjerg University Hospital, Copenhagen, Denmark. ${ }^{4}$ Center for Medical Research Methodology, University of Copenhagen, Copenhagen, Denmark.

Correspondence: Elisabeth Juul Gade, Dept of Respiratory Medicine, Bispebjerg University Hospital, Bispebjerg Bakke 23, DK-2400 Copenhagen NV, Denmark. E-mail: Elisabeth_gadedyahoo.com

ABSTRACT Evidence is increasing of an association between asthma and aspects of female reproduction. However, current knowledge is limited and furthermore relies on questionnaire studies or small populations. In a prospective observational cohort study to investigate whether time to pregnancy, the number of fertility treatments, and the number of successful pregnancies differ significantly between women with unexplained infertility with and without asthma.

245 women with unexplained infertility (aged 23-45 years) underwent questionnaires and asthma and allergy testing while undergoing fertility treatment. 96 women entering the study had either a former doctor's diagnosis of asthma or were diagnosed with asthma when included. After inclusion they were followed for a minimum of 12 months in fertility treatment, until they had a successful pregnancy, stopped treatment, or the observation ended.

The likelihood of achieving pregnancy was lower in women with asthma compared with those without asthma: median total time to pregnancy was 32.3 months in non-asthmatic women versus 55.6 months in those with asthma, hazard ratio 0.50 (95\% confidence interval $0.34-0.74) \mathrm{p}<0.001$.

Women with asthma had fewer successful pregnancies during fertility treatment, 39.6 versus $60.4 \%$ $(\mathrm{p}=0.002)$. Increasing age was of negative importance for expected time to pregnancy, especially among asthmatic women (interaction between age and asthma on time to pregnancy, $\mathrm{p}=0.001$ ). Female asthmatics had a longer time to pregnancy and less often became pregnant than non-asthmatic women. Increasing age reduced the chances of conceiving especially among asthmatic women. The causal relationship between asthma and subfertility remains unclear.

@ERSpublications

Asthma can be a cause of subfertility: increasing time to pregnancy and decreasing successful number of pregnancies http://ow.ly/WAE6b

A press release for this article is available from erj.ersjournals.com/press

Received: Aug 212015 | Accepted after revision: Nov 272015 | First published online: Feb 122016

This article has supplementary material available from erj.ersjournals.com

Clinical trials: This study is registered at ClinicalTrials.gov with identifier number NCT01788735.

Support statement: This study was supported by grants from: FAPS (Union of Practicing Specialists) grant number: 29241/11; Lundbeck Pharmaceutical grant number: R100-A9502; and the Medical Association Denmark. Funding information for this article has been deposited with FundRef.

Conflict of interest: None declared.

Copyright (CERS 2016 


\section{Introduction}

Several studies have shown a link between various aspects of female reproduction and asthma. Particularly, asthma has been associated with age at menarche, menstrual cycle, oral contraceptive use, pregnancy, menopause and hormone replacement therapy [1-6].

Moreover, asthmatic women of reproductive age are more likely than men with asthma to be admitted to hospital for an exacerbation and to have from more severe asthma [7-9].

Recently, a registry-based questionnaire study of twins demonstrated that asthma was associated with prolonged time to pregnancy (TTP) and thereby possibly impairs fertility [10]. Additionally, in a more recent study we showed that vascular endothelial growth factor (VEGF) is significantly decreased in the endometrial cavity in women with unexplained infertility and concomitant asthma compared to women without asthma [11]. This observation could partly explain these patients' reduced fertility, as high levels of VEGF in the uterus seem to support fertility in women without asthma [12].

This is further supported by a study by KäLLÉN et al. [13], which showed that a greater proportion of women who undergo fertility treatment use anti-asthmatic drugs. In contrast TATA et al. [14] found no evidence that fertility rates (live births per 1000 person years) among women with asthma are lower than among healthy women.

We therefore undertook a prospective clinical observational study with the hypothesis that time to pregnancy, number of fertility treatments, and number of successful pregnancies differ significantly between women with unexplained infertility with and without asthma.

\section{Methods}

\section{Patients}

Between December 2011 and December 2013, we recruited women with unexplained infertility (both heterosexual and lesbian) attending a Danish fertility clinic (Copenhagen Fertility Center, Copenhagen, Denmark) to participate in a clinical observational study with a minimum of 12 months of observations. The screening visit included an interview and objective tests for asthma and allergy.

We screened 420 women and included those with a diagnosis of unexplained infertility (12 months of timed sexual intercourse without pregnancy ( 6 months if aged $>35$ years)), open fallopian tubes, normal uterine anatomy, regular menstruations, normal s-luteinizing hormone, s-follicle stimulating hormone and s-thyroid stimulating hormone, and no male factor infertility), aged 18-45 years who had given informed consent. Singles and lesbians were diagnosed unexplained infertile if the above mentioned criteria were met except for male factor infertility and timed sexual intercourse. Instead singles and lesbians were included after six cycles of fertility treatment without a successful pregnancy. Those excluded were smokers and former smokers with $>10$ pack years, termination of fertility treatment directly after inclusion, a recent respiratory chest infection (within 14 days of screening), any systemic, gynaecological or respiratory diseases other than asthma. A total of 305 women fulfilled these criteria of which $250(82 \%)$ were included; 96 with and 149 without asthma. The remaining 55 women declined participation after introduction to the study (figure 1).

\section{Asthma diagnosis and treatment}

The diagnosis of asthma was based on symptoms clarified in the interview, such as shortness of breath, cough, chest tightness, wheezing and exercise-induced respiratory symptoms, as well as the asthma control

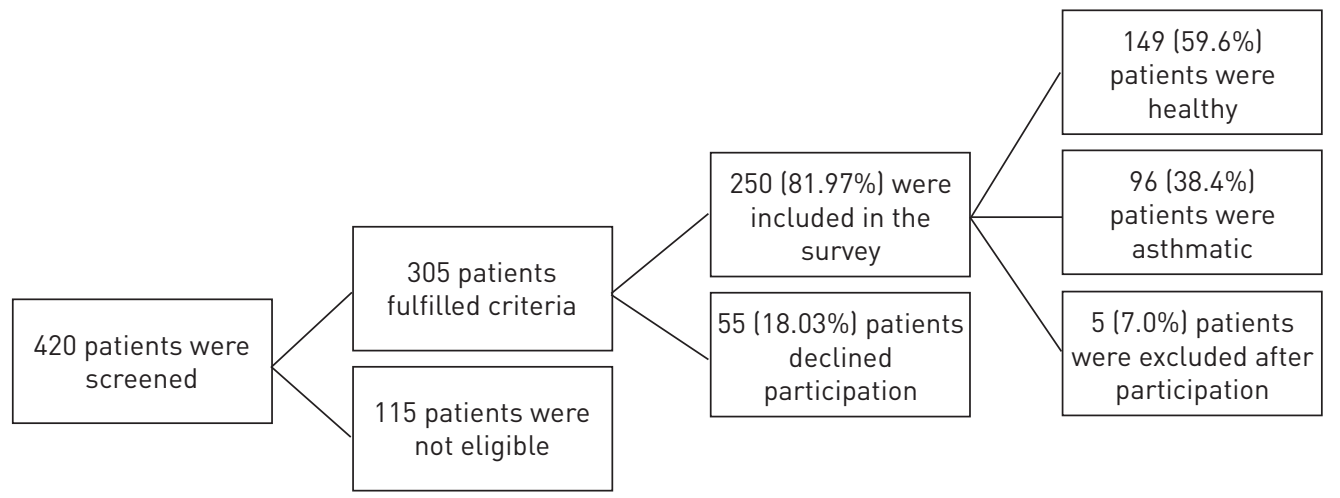

FIGURE 1 Flow diagraph showing selection process for participants of the study. 
questionnaire (ACQ) and Global Initiative for Asthma severity classification [15, 16]. Asthma was defined as former doctor-diagnosed asthma and/or as current respiratory symptoms consistent with asthma supported by forced expiratory volume in $1 \mathrm{~s}(\mathrm{FEV} 1)$ and a positive airway challenge test (metacholine provocative dose causing a $20 \%$ fall in FEV $1\left(\mathrm{PD}_{20}\right)<8 \mu \mathrm{mol}$ and/or mannitol PD15 $\leqslant 635 \mathrm{mg}$ ).

\section{Questionnaires}

The ACQ 7 was used to investigate self-estimated asthma symptoms during the week before the first visit [17]. ACQ is measured on a 7-point scale, where 0 is no symptoms and 6 is severe asthma symptoms. The total score was divided by 7 and a mean value was calculated.

\section{Lung function testing}

Spirometric measurements of FEV1 and forced vital capacity (FVC) were taken with the Easy One spirometer (ndd, Zurich, Switzerland). The FEV1 \% predicted was calculated. The ratio of the pre-provocation values of FEV1 and FVC was used to calculate the degree of airway obstruction.

\section{Bronchial challenge}

Methacholine challenge testing was performed on all patients using the method described by CRAPO et al. [18].

A cumulative dose of methacholine of $\leqslant 8 \mu \mathrm{mol}$ and a decrease in $\mathrm{FEV}_{1} \geqslant 20 \%$ were defined as having airway hyperresponsiveness (AHR).

Mannitol challenge testing was performed in women with a positive metacholine test and in all women with a former doctor's diagnosis of asthma, using dry powder of mannitol (Aridol), which was supplied in a single user kit (Pharmaxis Ltd, Frenchs Forest, Australia). A provocation concentration of $\leqslant 675 \mathrm{mg}$ and a decrease in FEV1 of $\geqslant 15 \%$ were defined as AHR.

For both challenge tests the response dose ratio was calculated as the percentage fall in FEV1 after the last dose, divided by the cumulative dose in milligrams.

\section{Exhaled nitric oxide fraction measurement}

Exhaled nitric oxide fraction $(F \mathrm{eNO}$ ) was measured using a chemiluminescence analyser (NIOX; Aerocrine $\mathrm{AB}$, Solna, Sweden). Measurements were taken for $10 \mathrm{~s}$ maintaining a constant flow rate of $50 \mathrm{~mL} \cdot \mathrm{s}^{-1}$ at each exhalation procedure. All measurements were performed in triplicate and the mean concentration was registered.

\section{Skin prick test}

Skin prick tests were performed using Soluprick (ALK, Copenhagen, Denmark) standardised allergens: birch, timothy grass, mugwort, horse, dog, cat, house dust mite (Der p1 and Der f2), Alternaria and Cladosporium. Atopy was defined as at least one positive test reaction with a mean of $\geqslant 3 \mathrm{~mm}$ greater than the negative control after $15 \mathrm{~min}$ and no dermographism.

\section{Blood sample analysis}

Whole blood samples were drawn from participants and incubated at room temperature for 30-45 min. Serum was isolated from whole blood by centrifugation, aliquoted into cryogenic tubes and stored at $-80^{\circ} \mathrm{C}$. The total concentration of IgE was quantified with ImmunoCAP (Thermo Fisher Scientific, Waltham, MA, USA). Serum concentrations of interleukin (IL)-6, IL-8 and tumour necrosis factor were assessed using Quantikine HS ELISA R\&D Kits (R\&D Systems, Minneapolis, MN, USA) (96-well custom cytokine/ chemokine assay). Duplicate measurements were performed and an average value of the two measurements was calculated. A difference between measurements of no more than $10 \%$ was considered acceptable.

Serum concentrations of high sensitivity of C-reactive protein (hsCRP) were assessed via Tina-quant hsCRP immunoturbidimetric assay using Roche/Hitachi MODULAR (Massachusetts, USA).

\section{Fertility treatment}

Treatments to establish pregnancy included stimulated cycles (timed intercourse), intrauterine inseminations (IUI), in vitro fertilisation and intracytoplasmic sperm injection (ICSI) using autologous or donated oocytes. ICSI and oocyte donation were used as alternative treatment, when no other treatments were successful. A detailed description of the treatment types is available in the supplementary material.

The semen quality after purification was obtained and semen motility was classified into four categories: immobile (0), non-progressive (1), slow (2), and progressive (3).

The fertility staff was unaware of the patients' asthma status while treating their infertility. Accordingly, the fertility treatment was independent of the participants' asthma status. 


\section{Outcomes}

The women were observed until pregnancy or for at least 1 year after inclusion, from their first cycle of fertility treatment, onwards until successful pregnancy, ending fertility treatment or the observation ended.

TTP in treatment was calculated from the first day of first treatment cycle until the first day in the last treatment cycle leading to successful pregnancy, termination or ended observation period. TTP was defined in three different ways: 1) Total TTP, the time from starting natural unprotected intercourse at home until successful pregnancy, termination or the observation period ended; 2) TTP in treatment, the time from the first treatment cycle until the last treatment cycle, termination or the observation period ended; and 3) TTP from inclusion, the time from the first treatment cycle after inclusion until the last treatment cycle leading to a successful pregnancy, termination, or the observation period ended.

Pregnancies were only included as successful pregnancies if resulting in live birth.

All fertility data; i.e. the time trying to conceive at home and in treatment, the number and type of fertility treatments and spontaneous abortions were determined by an initial interview, from medical records at Copenhagen Fertility Center and by a follow-up interview at the end of the study.

\section{Statistical analysis}

Data were analysed with the statistical package SPSS version 20.0 (IBM, North Castle, NY, USA). Mean and standard deviations were calculated for normally distributed data, whereas median and interquartile ranges (IQR) were used for skewed distributed data. The groups were compared using the two-sample t-test for the normally distributed continuous variables and the Mann-Whitney U-test for non-normally distributed continuous variables. Chi-square or Fisher's exact test were used for binary unpaired data. Kaplan-Meier survival plots and proportional hazards (Cox) regression were used to analyse differences in TTP between asthmatic and non-asthmatic women using the self-reported start time attempting pregnancy at home as the entry time and adjusting for confounders. Body mass index (BMI), age, former smoking, age at menarche, former pregnancies, number of fertility treatments at inclusion, semen quality and semen motility were selected as confounding variables due to their potential influence on asthma and TTP.

In sensitivity analyses Cox regression was also used to explore interactions between TTP and age, FEV1, BMI, atopic status, FeNO, AHR to metacholine and asthma of the partner. Values of $\mathrm{p}<0.05$ were considered significant.

Spontaneous pregnancies achieved between treatment cycles were included. Patients who achieved a successful pregnancy not leading to term birth were not censored at pregnancy but when the next successful pregnancy was achieved or treatment stopped.

\section{Ethics}

Approval was obtained from the local scientific ethics committee (project No.H-4-2011-118). Written and oral informed consent was obtained from all women before entering the study.

\section{Results}

Descriptive characteristics

The mean (range) age of the 245 patients was 35.8 (23-45) years and the mean \pm SD BMI was $23.8 \pm 4.4 \mathrm{~kg} \cdot \mathrm{m}^{-2}$ (table 1$)$.

After asthma testing and treatment evaluation 81 women had current asthma, of which 37 women were newly diagnosed, while 15 had former asthma. Of the 81 women with current asthma, 56 women were prescribed a combination of inhaled corticosteroids (ICS) and short-acting $\beta_{2}$ agonist (SABA) as a result of their moderate-to-severe asthma, while 25 women only received SABA due to their mild asthma.

FEV1 was significantly lower among those with asthma than among those without (2.98 versus $3.21 \mathrm{~L}$ ), $\mathrm{p}<0.001$. Serum IgE and eosinophilic cationic protein were significantly higher among women with asthma than among those without (38.9 versus $16.2 \mathrm{kU} \cdot \mathrm{L}^{-1}$ ) $\mathrm{p}<0.001$ and ( 1.0 versus $0.9 \mu \mathrm{g} \cdot \mathrm{L}^{-1}$ ) $\mathrm{p}=0.049$, respectively (table 1 ).

Semen motility ( 2.3 versus 2.2 ), $\mathrm{p}=0.034$ (table 2 ) was significantly higher in the partners of those with asthma.

\section{Main analyses}

The median total TTP was significantly increased among those with asthma: 55.6 versus 32.3 months (hazard ratio (HR) 0.50 (95\% CI 0.34-0.74), p<0.001) (table 2 and figure 2). The association remained significant after adjustment for age, BMI, former smoking, age at menarche, occurrence of former pregnancies, semen quality and motility and number of treatment cycles received in fertility treatment (HR 0.52 (95\% CI 0.35-0.77), $\mathrm{p}=0.003$ ). This association was also observed when only analysing TTP within fertility treatment (median 17.9 month in those without versus 35.6 month in those with asthma, HR 0.57 
TABLE 1 Characteristics of the study population

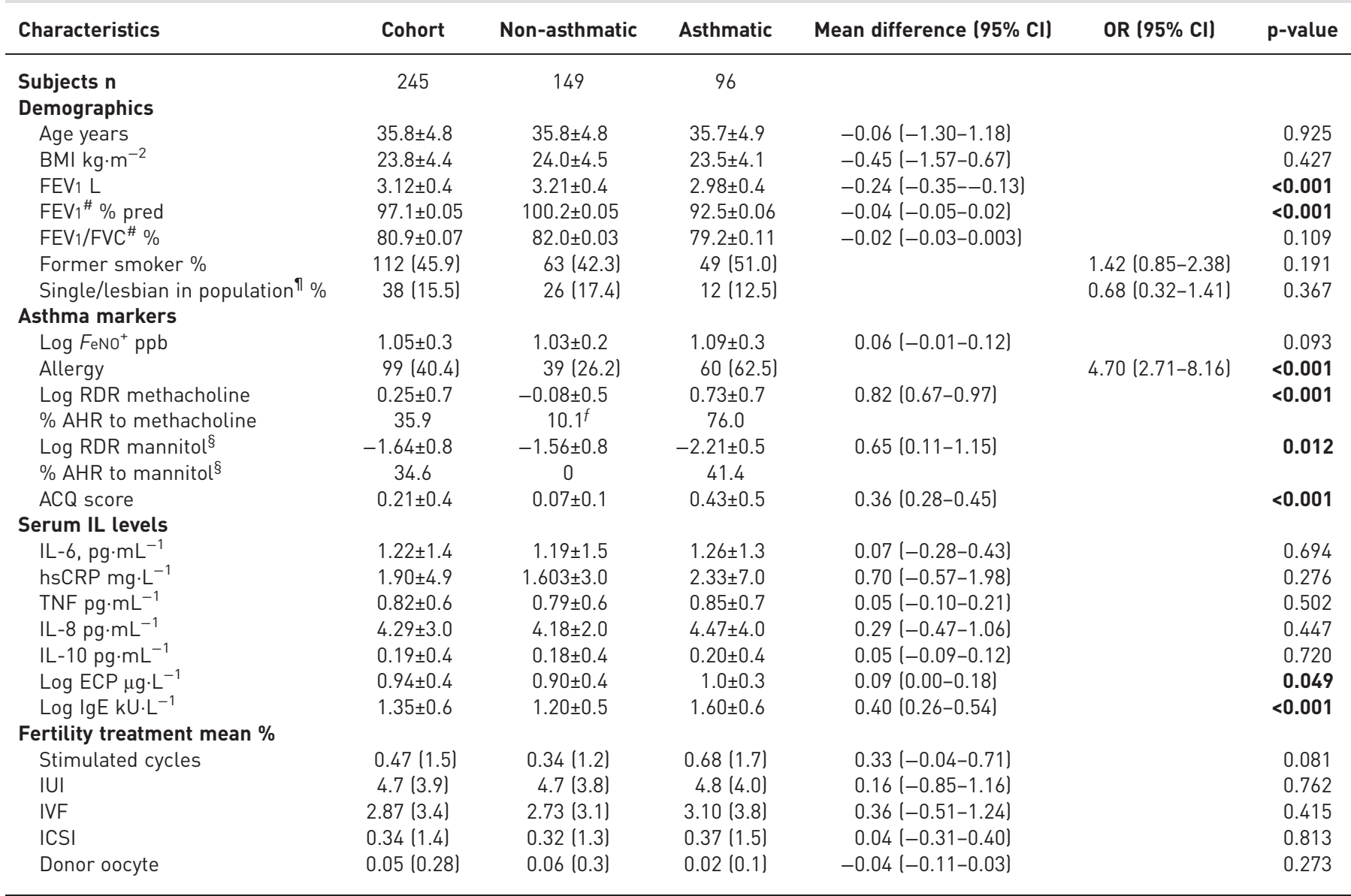

Data are presented as mean \pm SD or $\mathrm{n}(\%)$ unless otherwise stated. Data in bold is statistically significant. BMI: body mass index; FEV1: forced expiratory volume in $1 \mathrm{~s}$; FVC: forced vital capacity; $F$ eNO: exhaled nitric oxide fraction; RDR: response dose ratio; AHR: airway hyperresponsiveness; ACQ: asthma control questionnaire; IL: interleukin; hsCPR: high sensitivity C-reactive protein; TNF: tumour necrosis factor; ECP: eosinophil cationic protein; IUI: intrauterine insemination; IVF: in vitro fertilisation; ICSI: intra cytoplasmic sperm injection. \#: p-values and mean differences were calculated in log values; ${ }^{\text {9 }}$ : relationship was defined as single/lesbian or heterosexual; ${ }^{+}$: geometric mean; ${ }^{\S}$ : mannitol test was performed only on patients with AHR to methacholine; ${ }^{f}$ : all non-asthmatics with a post metacholine test had negative mannitol tests.

(95\% CI 0.39-0.84), $\mathrm{p}=0.004$ (table 2)), even after adjustment for confounders, which also included the time spent on trying to conceive before fertility treatment began, HR 0.66 (95\% CI 0.44-0.98), p=0.040. When using the date of clinical examination as the entry time instead, the results remained significant (HR 0.56 (95\% CI 0.38-0.81), $\mathrm{p}=0.002)$, even after adjustment for all mentioned confounders (HR 0.54 (95\% CI $0.36-0.80) \mathrm{p}=0.002$ ) (table 2). The estimated TTP for women with asthma was median (range) 13.5 (10.116.8) months versus 6.6 (2.9-10.4) months for those without asthma from inclusion.

When restricting the analysis to include only women with current asthma, the same tendency was observed for total TTP (HR 0.55 (95\% CI 0.36-0.83), p=0.004), after adjusting for confounders.

Among the 250 women, 128 (52.2\%) successful pregnancies occurred; 90 (60.4\%) among non-asthmatic women versus $38(39.6 \%)$ among asthmatic women, $\mathrm{p}=0.002$ (table 2).

\section{Sensitivity analyses}

Including an interaction term between age and asthma showed that older women with asthma were less likely to conceive than were their younger counterparts, $\mathrm{p}=0.001$. This effect was probably because women with asthma aged $\geqslant 35$ years were less likely to conceive than were women with asthma aged $<35$ years (25.9 versus $61.4 \%, \mathrm{p}<0.001$ ). Conversely, the pregnancy rates among non-asthmatic women aged $\geqslant 35$ years and $<35$ years were 56.7 and $68.6 \%$, respectively. The corresponding total TTPs among these four groups of women were: asthmatic women aged $\geqslant 35$ years 125.3 months, asthmatic women aged $<35$ years 33.2 months, non-asthmatic women aged $\geqslant 35$ years 38.3 months, and non-asthmatic women aged $<35$ years 26.3 months. 
TABLE 2 Reproductive characteristics

Cohort Non-asthmatic Asthmatic $\begin{gathered}\text { Mean difference } \\ (95 \% \mathrm{CI})\end{gathered} \quad$ HR $(95 \% \mathrm{Cl}) \quad$ OR $(95 \% \mathrm{CI}) \quad \mathrm{p}$-value

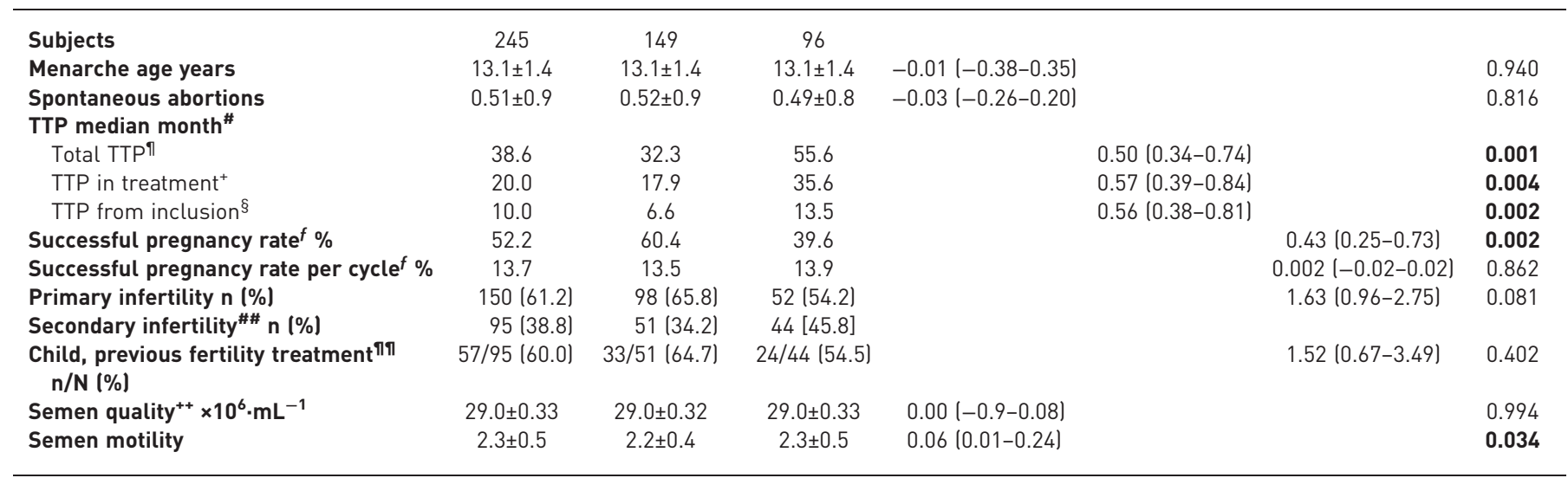

Data are presented as $\mathrm{n}$ or mean $\pm \mathrm{SD}$, unless otherwise stated. Data in bold is statistically significant. * : the results for total time to pregnancy (TTP) in total, in treatment and from inclusion were adjusted for confounders; ": total TTP from trying to conceive naturally to successful pregnancy in fertility treatment or stopping treatment; ${ }^{+}$: TTP treatment from the first until the last treatment cycle in fertility treatment; ${ }^{\S}$ : TTP from inclusion in the study until the last treatment cycle in the month; ${ }^{f}$ : successful pregnancy rate and successful pregnancy rate per treatment cycle occurring during the treatment period from December 2011 to December 2014; \#\# : had a child before participating in the study; "१?: conception of first child after fertility treatment, the percentages describe the amount of secondary infertility who had fertility treatment to conceive their first child; ${ }^{++}$: after purification (calculated in log).

There was no statistically significant interaction between asthma and BMI, $\mathrm{p}=0.970$; FEV1, $\mathrm{p}=0.150$; atopic status, $\mathrm{p}=0.376$; AHR, $\mathrm{p}=0.723$; asthma of the partner, $\mathrm{p}=0.568$; and FeNO, $\mathrm{p}=0.438$, regarding likelihood of conceiving.

There was no significant difference in TTP between those with treated and untreated asthma at the end of the observation period (HR 1.13 (95\% CI 0.60-2.15), p=0.706). Furthermore there was no age difference between the asthmatic women who were taking ICS versus those only treated with SABA (35.9 versus 35.5 years, $\mathrm{p}=0.707)$.

The fraction achieving pregnancy in the observation period (both those with and without asthma) differed from the group not achieving pregnancy regarding only age, i.e. they were significantly older (36.9 versus 34.6 years), $\mathrm{p}<0.001$.

FIGURE 2 Kaplan-Meier plot comparing the cumulative pregnancy rate $(\%)$ over time (months) in unexplained infertile women versus unexplained infertile asthmatic women, $p<0.001$.

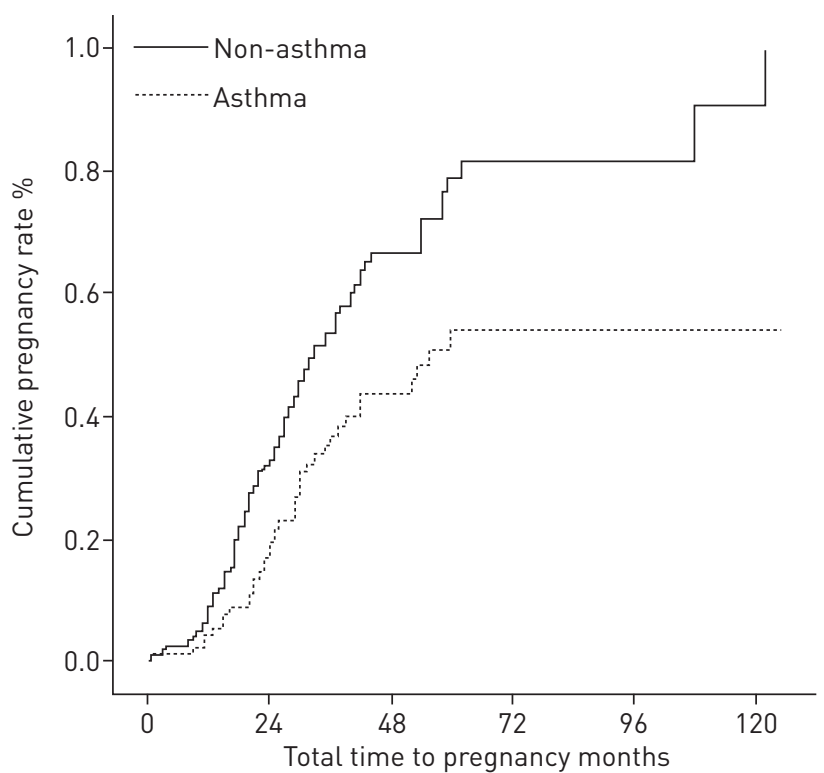


There was no difference in the reasons for censoring (i.e. dropping out of fertility treatment before the observation period ended either due to personal reasons or age above 45 years) in the Kaplan-Meier statistics between the non-pregnant women in the asthmatic and non-asthmatic group $(\mathrm{p}=0.731)$.

The fraction of asthmatic women achieving pregnancy in the observation period differed from the asthmatic group not achieving pregnancy regarding age (33 versus 37.5 years, $\mathrm{p}<0.001$ ), total treatment cycles $(7.2$ versus $10.1, \mathrm{p}=0.01)$, semen motility $(2.2$ versus $2.4, \mathrm{p}=0.034)$ and age at menarche $(12.8$ versus 13.4 years, $\mathrm{p}=0.031)$. The same tendency among the non-asthmatic cohort for conceiving and not conceiving was shown with regards to age (35.2 versus 36.8 years, $\mathrm{p}=0.042$ ), total treatment cycles ( 7.4 versus $9.3, \mathrm{p}=0.018)$ and semen motility $(2.1$ versus $2.3, \mathrm{p}=0.030)$. However, no difference in age at menarche was seen between the non-asthmatic women conceiving and the non-asthmatic women who did not conceive (13.3 versus 13.1 years, $\mathrm{p}=0.523$ ).

\section{Discussion}

This study showed that the likelihood of achieving pregnancy among women with unexplained infertility was reduced in individuals with asthma compared to those without asthma, even after adjusting for confounders. The impact of asthma on TTP was consistent in both older and younger individuals, with an increasing tendency with age. Furthermore asthma control and severity was not related to conceiving.

Our result showed that increasing age is of negative importance for expected TTP and pregnancy rate of all women, but particularly for women with asthma. This supports previous findings by TATA et al. [14] and GADE et al. [10] that showed women with asthma have lower fertility rates when older compared with similarly aged women without asthma. These findings could indicate that the severity, the disease progression and the years exposed to low-grade systemic inflammation, have an influence on fertility. However no interaction between asthma control, severity markers of asthma (FEV1, FeNO and AHR to metacholine) or any of the biomarkers and the likelihood of conceiving was found.

The increasing tendency with age among asthmatic women could also be related to the consumption of more asthma medication and reflect that a greater proportion of older asthmatic women receive ICS, due to more severe asthma. However, this was not the case as there was no age difference between those receiving ICS and SABA. Furthermore, there was no difference in TTP between the ICS- and the SABA-treated asthma patients. The medication for asthma does, therefore, not account for the difference either in regards to age or fertility.

Another possible explanation for our findings is a relationship between asthma and female sex hormones, as age of menarche was significantly higher among non-conceiving asthmatic women. This is surprising, since asthma normally is associated with early menarche. Later onset of menarche could impair these women's fertility, as it has been associated with increased risk of infertility and lower levels of anti-Müllerian hormone (lower ovarian reserve) [19, 20]. However, data are conflicting, and others show either no association or the opposite tendency [21].

The important strength of this study is the objectively supported asthma diagnoses and that the recall bias regarding TTP in treatment is minimal, as participants both when entering and terminating the study provided the information and their medical records supported this information. However, TTP when having timed sexual intercourse at home could be biased by recall problems, as medical records did not provide this information.

Another strength is the long follow-up time (maximum follow-up time was 125 months) and the power of the statistical method (survival analysis), which reduces spurious overestimation of fertility due to early drop-out of women who did not conceive.

A limitation of this study is that women who were willing and motivated to participate may differ significantly from the overall population. This could have biased the results, if women who denied participation either were non-asthmatic women with a long TTP or asthmatic women with a short TTP. However, the overall tendency seemed to be that many non-asthmatic women with a long fertility history participated hoping to find a reason for their infertility. Furthermore, a large amount of women (37 subjects) were diagnosed with asthma when entering study, which made the outcome unpredictable.

Other limitations could be an underestimation of the complexity and heterogeneity of fertility patients in fertility treatments and that it was a one centre study. Socioeconomic status and lack of finances to pay for treatment might be a factor, especially for those aged $>40$ years. Furthermore couples might voluntarily decide to wait months before continuing treatment. Two women (one asthmatic and one non-asthmatic) got pregnant naturally in one of those waiting periods and were included in the analysis as successful pregnancies. However those factors should be equally distributed between groups. 
Finally, current and former asthma patients were mixed, which could make the asthma group less homogeneous. However, former asthma patients were included in the asthma group as they could not be included as non-asthma and as it is unknown if suffering from asthma at some point can affect the ovaries and thereby, fertility.

In conclusion, asthma has a negative effect on female fertility in terms of likelihood of conceiving and successful pregnancies; an effect that increases with age and seems unaffected by treatment for asthma. In a future perspective it seems important that studies attempt to explore the more specific causes of our observations to be able to counteract the negative effect of asthma on fertility.

\section{Acknowledgements}

A special thanks to Jan Blaabjerg, Marianne Kornerup and the wonderful staff at Copenhagen Fertility Center (Copenhagen, Denmark) for their dedication, support and help in collecting participants for the project.

\section{References}

1 Macsali F, Real FG, Plana E, et al. Early age at menarche, lung function, and adult asthma. Am J Respir Crit Care Med 2011; 183: 8-14.

2 Galobardes B, Patel S, Henderson J, et al. The association between irregular menstruations and acne with asthma and atopy phenotypes. Am J Epidemiol 2012; 176: 733-737.

3 Macsali F, Real FG, Omenanas ER, et al. Oral contraception, body mass index, and asthma: a cross-sectional Nordic-Baltic population survey. J Allergy Clin Immunol 2009; 123: 391-339.

4 Murphy V, Gibson PG. Asthma in pregnancy. Clin Chest Med 2011; 32: 93-110.

5 Foschino Barbaro MP, Palladino VR, Carpagnano O, et al. Menopausal asthma: a new biological phenotype? Allergy 2010; 65: 1306-1312.

6 Real FG, Svanes C, Omenaas E. Hormone replacement therapy, body mass index and asthma in perimenopausal women: a cross sectional survey. Thorax 2006; 61: 34-40.

7 Schatz M, Dombrowski MP, Wise R, et al. Asthma morbidity during pregnancy can be predicted by severity classification. J Allergy Clin Immunol 2003; 112: 283-288.

8 Rao CK, Moore CG, Bleecker E, et al. Characteristics of perimenstrual asthma and its relation to asthma severity and control-data from the Severe Asthma Research Program. Chest 2013; 143: 984-992.

9 Trawick DR, Holm C, Wirth J. Influence of gender on rates of hospitalization, hospital course, and hypercapnea in high-risk patients admitted for asthma: a 10-year retrospective study at Yale-New Haven Hospital. Chest 2001; 119: 115-119.

10 Gade EJ, Thomsen SF, Lindenberg S, et al. Asthma affects time to pregnancy and fertility: a register-based twin study. Eur Respir J 2014; 43: 1077-1085.

11 Gade EJ, Thomsen SF, Lindenberg S, et al. Lower values of VEGF in endometrial secretion are a possible cause of subfertility in asthmatic patients. J Asthma 2015; 52: 366-342.

12 Seo WS, Jee BC, Moon SY. Expression of endometrial protein markers in infertile women and the association with subsequent in vitro fertilization outcome. Fertil Steril 2011; 95: 2707-2710.

13 Källén B, Olausson PO. Use of anti-asthmatic drug during pregnancy. 1. Maternal characteristics, pregnancy and delivery complications. Eur J Clin Pharmacol 2007; 63: 363-373.

14 Tata LJ, Hubbard RB, McKeever TM, et al. Fertility rates in women with asthma, eczema, and hay fever: a general population-based cohort study. Am J Epidemiol 2007; 165: 1023-1030.

15 Masoli M, Fabian D, Holt S, et al. The global burden of asthma: executive summary of the GINA Dissemination Committee report. Allergy 2004; 59: 469-478.

16 Bousquet J. Global initiative for asthma (GINA) and its objectives. Clin Exp Allergy 2000; 30: Suppl 1, 2-5.

17 Juniper EF, Bousquet J, Abetz L, et al. Identifying 'well-controlled' and 'not well-controlled' asthma using the Asthma Control Questionnaire. Respir Med 2006; 100: 616-621.

18 Crapo RO, Casaburi R, Coates AL, et al. Guidelines for methacholine and exercise challenge testing-1999. Am J Respir Crit Care Med 2000; 161: 309-329.

19 Guldbrandsen K, Håkonsen LB, Ernst A, et al. Age at menarche and time to pregnancy. Hum Reprod 2014; 29: 2058-2064.

20 Braggs JM, Kuzawa CW, Agustin SS, et al. Age at menarche and parity are independenly associated with anti-Müllerian hormone, a marker of ovarian reserve, in a Filipino young adult women. Am J Hum Biol 2012; 24: 739-745.

21 Weghofer A, Kim A, Barad DH, et al. Age at menarche: a predictor of diminished ovarian function? Fertil Steril 2013; 100: 1039-1043. 